\title{
Consenso y Disenso en la Memoria Histórica y en las Actitudes Hacia la Reparación en Tres Generaciones de Chilenos
}

\section{Consensus and Dissent in Historical Memory and in Attitudes Toward Reparation in Three Generations of Chileans}

\author{
Héctor Carvacho \\ Universität Bielefeld \\ Jorge Manzi, Andrés Haye, Roberto González y Marcela Cornejo
Pontificia Universidad Católica de Chile
}

\begin{abstract}
En 2 estudios correlacionales, con muestras basadas en cuotas de nivel socioeconómico, edad y género $\left(N_{1}=996\right.$ y $\left.N_{2}=841\right)$, en Santiago de Chile se evaluaron 2 hipótesis: (a) chilenos que se socializaron políticamente en diferentes épocas (previa, durante y posterior a la dictadura) y que tienen diferente orientación política (izquierda, centro o derecha) manifiestan consenso en su memoria histórica, pero disenso en su valoración de las políticas de reparación hacia víctimas de la dictadura y (b) las actitudes ideológicas (autoritarismo, apoyo a la democracia y orientación a la dominancia social) explican las diferencias en la valoración de las políticas de reparación. El análisis de una pregunta abierta muestra consenso intergeneracional y entre grupos políticos en que el golpe de Estado de 1973 y la transición a la democracia son los elementos centrales de la historia política chilena. Usando un modelo de ecuaciones estructurales, se encontró que los grupos políticos disienten en su valoración de las políticas de reparación (la izquierda tiene actitudes más positivas), en función de las actitudes ideológicas que subyacen a la orientación política.
\end{abstract}

Palabras clave: generaciones, memoria histórica, socialización política, orientación política, actitudes ideológicas

\begin{abstract}
In 2 correlational studies conducted in Santiago de Chile $\left(N_{1}=996\right.$ and $\left.N_{2}=841\right)$-with samples based on quotas of socioeconomic status, age, and gender -2 hypotheses were tested: (a) that Chileans socialized in different political context (before, during or after the dictatorship) and who have different political orientations (left, center or right) exhibit consensus in their historical memory, but differ concerning the evaluation of reparation policies toward victims of the dictatorship, and (b) that ideological attitudes (authoritarianism, support for democracy, and orientation towards social dominance) explain differences in the evaluation of the reparation policies. Analysis of an open question showed consensus between generations and between political groups about considering the coup d'état in 1973 and the transition to democracy as the central elements of Chilean political history. Results of structural equation modeling showed that political groups differ in their evaluation of reparation policies (with the left being more supportive). Dissent was explained by the ideological attitudes underlying the left-right distinction.
\end{abstract}

Keywords: generations, historical memory, political socialization, political orientation, ideological attitudes

Héctor Carvacho, Institut für interdisziplinäre Konflikt- und Gewaltforschung, Universität Bielefeld, Alemania; Jorge Manzi, Andrés Haye, Roberto González y Marcela Cornejo, Escuela de Psicología, Pontificia Universidad Católica de Chile, Santiago, Chile.

Esta investigación fue financiada por el proyecto FONDECYT N ${ }^{\circ} 1050887$ y por el Centro Interdisciplinario de Estudios Interculturales e Indígenas, CONICYT/FONDAP N 15110006.

La correspondencia relativa a este artículo debe ser dirigida a Héctor Carvacho, Institut für interdisziplinäre Konflikt- und Gewaltforschung, Universität Bielefeld, Universitäetsstraße 25, 33615 Bielefeld, Alemania. E-mail: hector.carvacho@uni-bielefeld.de 
¿Qué tan profundo es el impacto de los eventos políticos más importantes de la historia en la cultura política de un país? ¿Es posible hablar de generaciones que son definidas por su experiencia compartida en torno a uno de estos eventos? Durante los últimos 100 años no hubo un evento más relevante para la historia política chilena que el golpe de Estado de 1973 y la subsecuente dictadura que duró 17 años. A través de dos estudios, buscamos, en primer lugar, explorar el impacto que tuvo la dictadura en la cultura política chilena y, en particular, si la memoria es afectada por el contexto político en el que las personas han sido socializadas: antes, durante o después de la dictadura. En segundo lugar, examinamos la relación entre las actitudes sociales que subyacen la orientación política y las actitudes sobre las políticas de reparación hacia las víctimas de violaciones de los derechos humanos.

La relevancia del golpe de Estado del 11 de Septiembre de 1973 y la dictadura encabezada por Pinochet hasta Marzo de 1990 se refleja en el estudio de la memoria histórica en Chile. Esta línea de investigación se ha concentrado en identificar cómo la memoria histórica se construye usando el golpe de Estado y la dictadura como sus referentes más importantes (e.g., Arnoso, Cárdenas \& Páez, 2012; Escobar \& Fernández, 2008; Haye, 2003; Manzi, Helsper, Ruiz, Krause \& Kronmüller, 2003; Piper, 2005; Prado \& Krause, 2004; Tocornal, 2008; Waldman, 2009). Se han descrito, por un lado, los patrones discursivos de los distintos grupos ideológicos involucrados en el conflicto (e.g., Manzi et al., 2004; Ruiz \& Krause, 2003) y, por otro, las diferencias entre generaciones que fueron socializadas en distintos contextos políticos (Arnoso et al., 2012; Concha, Guichard \& Henríquez, 2009; Guichard \& Henríquez, 2011; Manzi et al., 2003).

En los estudios que reportamos en este artículo buscamos extender esta línea de investigación, abordando, en primer lugar, los aspectos consensuales de la memoria intergeneracional y, en segundo lugar, los elementos ideológicos que subyacen al disenso entre grupos ideológicos.

\section{Memoria y Generaciones Políticas}

El concepto de generación política viene de la tradición de la sociología del conocimiento y fue usado inicialmente por Karl Mannheim (1952), quien postuló que las condiciones socio-históricas en que las personas viven definen sus posibilidades de conocimiento. De este modo, un grupo de personas que comparten un cierto tiempo o época deberá estar marcado por las condiciones socio-históricas específicas de ese tiempo. Este grupo corresponde a una generación.

El desarrollo posterior del concepto fue influido por la psicología del desarrollo. La discusión se centró en vincular el desarrollo psicológico individual con los procesos socio-históricos desde dos perspectivas dominantes. La primera, más prominente en la sociología, se centra en el concepto de trayectorias individuales de vida (Mayer, 2009), observando el impacto en ellas de las estructuras macrosociales y de las instituciones. La segunda ha predominado en la psicología y se basa en el concepto de ciclo vital (Baltes, 1987; Baltes, Staudinger \& Lindenberger, 1999), reconociendo una multiplicidad de elementos que convergen para explicar los procesos de cambio: factores del desarrollo; dinámicas de crecimiento y ganancia, por un lado, y deterioro y pérdida, por otro; factores históricos y otros elementos de la estructura sociocultural; plasticidad en el desarrollo y procesos de adaptación.

Desde la perspectiva psicológica, se ha derivado la hipótesis de los años impresionables, que subyace a la idea de generaciones políticas (Alwin \& Krosnick, 1991; Osborne, Sears \& Valentino, 2011; Sears \& Valentino, 1997; Valentino \& Sears, 1998). Esta hipótesis plantea que el final de la adolescencia y el comienzo de la adultez joven constituyen etapas en que las personas son especialmente susceptibles de ser impactadas por su entorno socio-histórico. Como resultado, las experiencias políticas que son vividas en estas etapas del ciclo vital 
juegan un rol central en la consolidación de las configuraciones ideológicas, incluida la memoria, y permanecerán estables por el resto de la vida.

El estudio de las generaciones políticas ha sido de central importancia para la investigación sobre memoria histórica. La proximidad de eventos históricos relevantes a los años impresionables deja una marca importante en la forma en que las personas construyen su memoria, siendo estos eventos recordados y catalogados como los más importantes para la sociedad (Schuman \& Corning, 2006; Schuman \& Rodgers, 2004; Schuman \& Scott, 1989).

En el caso chileno, las investigaciones en el tema han mostrado resultados mixtos. Por un lado, cuando se ha preguntado por los eventos más importantes de la historia, los resultados muestran un consenso en torno a la centralidad del golpe de Estado entre generaciones socializadas antes, durante y después de la dictadura (Manzi et al., 2003). Estos resultados llevaron a Manzi et al. (2003) a concluir que, a pesar de la existencia de un componente generacional, la prominencia del golpe para la memoria histórica chilena es tal que sobrepasa las diferencias generacionales. Por otro lado, en un estudio más reciente, en el que los participantes debían mencionar un evento que hubiese ocurrido durante el transcurso de sus vidas, los resultados mostraron que el golpe de Estado es central para quienes lo vivieron en sus años impresionables o después, pero para quienes lo vivieron a muy temprana edad la vuelta a la democracia cobra mayor relevancia (Concha et al., 2009; Guichard \& Henríquez, 2011). Esta idea es concordante con la de la existencia de una nueva generación posterior al plebiscito que puso fin a la dictadura (Toro, 2008). Tanto el estudio de Manzi et al. (2003) como el de Guichard y Henríquez (2001 presentan limitaciones que deben ser abordadas. Una de las limitaciones del estudio de Manzi et al. (2003) es que se llevó a cabo en un periodo en que la figura de Pinochet estaba en el centro de la discusión pública, pues en esa época había sido detenido en Londres por el juicio que se llevaba en su contra por crímenes contra la humanidad. Esto pudo haber hecho saliente los eventos conectados con la dictadura, causando que el nivel de consenso encontrado fuera mayor (ver Jelin, 2001). Se vuelve relevante, entonces, replicar el estudio en un momento en que no haya una contingencia noticiosa que influya directamente en los resultados. El estudio de Guichard y Henríquez (2011), por su parte, solo permitió identificar hechos que ocurrieron durante la vida de los entrevistados, por lo que la valoración del golpe de Estado por parte de las generaciones jóvenes no se exploró. A pesar de que el golpe de Estado es un evento que los jóvenes no vivieron personalmente, la transmisión intergeneracional podría haberlo convertido en el evento más relevante pare ellos.

\section{Memoria e Ideología}

Desde Halbwachs (1925) y Bartlett (1932), la memoria histórica se concibe como una dimensión social y una construcción colectiva. Más aún, en los últimos años se ha mostrado cómo los procesos de categorización e identificación grupal tienen un impacto directo en la memoria (Haye, 2003; Sahdra \& Ross, 2007). Haye (2003) mostró experimentalmente que la identidad grupal afecta el juicio de verdad respecto de un recuerdo y los recuerdos, a su vez, afectan los procesos de categorización grupal. El estudio se centró en el caso chileno y mostró cómo las identidades políticas de izquierda y derecha (a nivel grupal) son centrales para la formación de memoria específica que se construye en relación con estas identidades.

Los hallazgos de dicho estudio son concordantes con otros que, usando diferentes metodologías, han mostrado también que el relato histórico depende de la identidad política de los sujetos (Manzi et al., 2003, 2004; Piper, 2005; Ruiz \& Krause, 2003; Tocornal, 2008; Waldman, 2009). De estos estudios se concluye que, a pesar del consenso respecto de cuáles son los eventos más importantes, existe un notable disenso respecto de la interpretación de las causas y consecuencias del golpe y la dictadura, que depende de las orientaciones políticas. 
El estudio de las consecuencias de la dictadura ha abordado múltiples elementos. Entre ellos destaca la transmisión, no solo de la memoria a las nuevas generaciones, sino también de la experiencia de traumatización, tanto de víctimas directamente afectadas por la represión como de sus familiares y cercanos y de personas que han estado en contacto con la experiencia traumática represiva (Becker \& Díaz, 1998; Cornejo, Brackelaire \& Mendoza, 2009; Cornejo, Morales, Kovalskys \& Sharim, 2013; Faúndez \& Cornejo, 2010; Kovalskys \& Morales, 2001; Lira, Becker \& Castillo, 1991; Lira \& Castillo, 1991; Morales \& Cornejo, 2013; Sharim, Kovalskys, Morales \& Cornejo, 2011). Sin embargo, el ámbito en el que el disenso basado en identidad política ha sido más evidente es el de las políticas de reparación (Cornejo et al., 2007; Lira \& Morales, 2005; Montenegro \& Piper, 2009; Reyes, 2007; Ruiz \& Krause, 2003). Se ha mostrado que las políticas que buscan la reconciliación son percibidas controversialmente. Las instancias más importantes sobre verdad y reconciliación que ha llevado a cabo el Estado chileno han sido la Comisión Nacional de Verdad y Reconciliación en 1990, la Comisión Nacional Sobre Prisión Política y Tortura en 2003, la Mesa de Diálogo en 2001 y la Comisión Asesora Presidencial Para la Calificación de Detenidos Desaparecidos, Ejecutados Políticos y Víctimas de Prisión Política y Tortura en 2009 (Cárdenas, Páez \& Rimé, 2013; Cornejo et al., 2007; Cornejo, Rojas \& Mendoza, 2009; Lira \& Morales, 2005). Mientras las personas de izquierda consideran que el enjuiciamiento de los responsables por las violaciones de los derechos humanos, el reconocimiento público del daño causado y la reparación hacia las víctimas son condiciones necesarias para la reconciliación, las personas de derecha consideran que la unidad nacional conseguida a través del perdón de las víctimas es lo más importante pare la reconciliación (Ruiz \& Krause, 2003). Estudios con jóvenes universitarios, muy distantes biográficamente con los hechos del golpe de Estado, muestran también importantes diferencias entre quienes se identifican con la derecha y la izquierda en la propensión a reparar y a perdonar a la otra parte (ver González, Manzi \& Noor, en este número).

A pesar de los intentos por describir la distinción izquierda-derecha como menos relevante en los últimos años, se ha mostrado que esta sigue siendo una categoría fundamental en la que la mayoría de las personas se puede auto-clasificar (Jost, 2006), lo cual también aplica para el contexto latinoamericano y Chile en particular (Colomer \& Escatel, 2005). A la distinción izquierda-derecha subyace una serie de actitudes ideológicas que se configuran de forma consistente en diversos contextos (Carvacho \& Haye, 2008; Haye, Carvacho, González, Manzi \& Segovia, 2009). Dos parecen ser las actitudes más importantes para entender la diferencia: preferencia por cambio versus statu quo y apoyo a la igualdad (Jost, Glaser, Kruglanski \& Sulloway, 2003). Específicamente, las personas de izquierda tienden a preferir el cambio social, propiciando una organización más igualitaria de la sociedad, a rechazar actitudes nacionalistas y autoritarias y a mostrar apoyo al régimen democrático. Por el contrario, las personas de derecha muestran preferencia por las tradiciones, la preservación del orden actual de las cosas y las jerarquías sociales. Estas personas tienden a identificarse más con su grupo nacional, muestran más actitudes patrióticas y nacionalistas y cuidan más la cohesión grupal a través de actitudes autoritarias.

En el presente estudio nos enfocamos en tres actitudes ideológicas: autoritarismo de derecha, orientación a la dominancia social y apoyo a la democracia. El autoritarismo de derecha incluye tres dimensiones: (a) agresión autoritaria, que se define como el apoyo a autoridades fuertes y punitivas; (b) sumisión autoritaria, definida como la obediencia irreflexiva a las autoridades y (c) convencionalismo, que es una preferencia por lo previamente existente y un apego estricto a las normas grupales (Altemeyer, 1981). La orientación a la dominancia social es una disposición individual a apoyar y naturalizar las jerarquías sociales basadas en grupos arbitrarios (Pratto, Sidanius, Stallworth \& Malle, 1994; Sidanius \& Pratto, 1999, 2012). Por último, el apoyo a la democracia se define como la preferencia de regímenes democráticos por sobre regímenes autoritarios o la indiferencia respecto del tema (Carvacho \& Haye, 2008). Las tres actitudes subyacen a la distinción 
entre izquierda y derecha, tanto en Chile como internacionalmente (Haye et al., 2009; Jost et al., 2003; Jost, Federico \& Napier, 2009). Es más, se ha propuesto el concepto de configuración ideológica para entender el modo en que estas actitudes se organizan (Carvacho, 2010; Carvacho \& Haye, 2008). Esta noción supone que estas actitudes son relativamente estables y son relevantes para entender cómo un sujeto toma posición frente a objetos del mundo político. En culturas en las que el mundo político se organiza en torno a un eje, como izquierda y derecha, estas actitudes pueden ser entendidas como una unidad que se ordena en torno a un núcleo que implica intolerancia y derogación de otros (Carvacho, 2010), donde niveles más altos de estas actitudes (y más bajos para el caso de apoyo a la democracia) significa más derechismo (Haye et al., 2009).

Las actitudes ideológicas usadas en nuestro estudio son antecedentes de una multiplicidad de actitudes sociales, tales como prejuicio, etnocentrismo y religiosidad (Ho et al., 2012; Mavor, Louis \& Laythe, 2011; McFarland, 2010a; Sidanius \& Pratto, 2012). Sin embargo, para nuestra investigación fueron de especial interés los resultados de un estudio que mostró que estas actitudes predicen el apoyo a los derechos humanos y su implementación (McFarland, 2010b).

\section{La Presente Investigación}

Presentamos dos estudios transversales y correlacionales que tuvieron como objetivo mostrar, primero, cómo el trabajo de memoria es usado para definir un campo discursivo consensuado y, segundo, que al interior de este campo surgen los procesos de diferenciación ideológica. En el primer estudio exploramos el recuerdo libre, permitiendo a los participantes identificar aquellos eventos de la historia política chilena que ellos consideran los más importantes. De forma concordante con algunos estudios previos en el tema (e.g., Manzi et al., 2003), hipotetizamos que los participantes identifican de forma consensual los eventos más importantes de la historia política chilena, a saber, el golpe de Estado de 1973, la dictadura y la transición a la democracia.

En el segundo estudio exploramos de forma directa un tema que es materia de disenso: las políticas de memoria y reparación hacia las víctimas de la dictadura. Hipotetizamos que los mismos participantes - que en el primer estudio tendrían una mirada consensual en torno a los eventos más importantes- difieren en su posición respecto de las políticas de reparación y memoria. El disenso sería explicado por el trasfondo ideológico del conflicto, vale decir, por las actitudes ideológicas que subyacen a la distinción entre izquierda y derecha.

Ambos estudios dan cuenta del campo compartido en el que las generaciones y los grupos políticos se juegan su posicionamiento y, al mismo tiempo, de ámbitos específicos de diferenciación en la cultura política chilena, en este caso, sobre las actitudes hacia la reparación. En otras palabras, en el marco de la discusión en torno a los conflictos político-ideológicos en la historia reciente de Chile, con estos estudios buscamos identificar cómo el consenso y el disenso trabajan de forma complementaria para dar forma a la cultura política chilena.

\section{Estudio 1}

\section{Método}

Participantes. El año 2005, en la ciudad de Santiago, Chile, 996 participantes fueron contactados para tomar parte en un estudio más amplio sobre cultura política. El estudio consistió en la aplicación de un cuestionario que incorporó múltiples temas respecto del desarrollo de actitudes políticas, valores, confianza en instituciones, entre otros (e.g., Haye et al., 2009). 
La selección de los participantes se hizo en dos etapas. Primero, con base en la caracterización socioeconómica del censo de 2002, se seleccionó aleatoriamente un grupo de manzanas de entre todas las manzanas de Santiago, donde tres niveles socioeconómicos - alto, medio y bajo - fueron igualmente representados. En la segunda etapa, cuotas de cinco personas, basadas en sexo y edad de los participantes, fueron aleatoriamente seleccionadas al interior de cada manzana. La muestra estuvo compuesta por participantes de tres grupos etarios: (a) personas entre 50 y 60 años, expuestas al golpe de Estado durante sus años impresionables; (b) entre 33 y 40 años, para quienes el retorno a la democracia coincidió con sus años impresionables y (c) entre 17 y 21 años, quienes estaban en sus años impresionables al momento de la entrevista.

La muestra estuvo compuesta por 479 (48\%) hombres y 516 (52\%) mujeres (un caso sin información). Respecto del grupo generacional, 341 (34\%) pertenecen a la generación menor, $326(33 \%)$ a la media y 329 (33\%) a la mayor. Respecto del grupo socioeconómico, 263 (27\%) son de nivel alto, 356 (37\%) del medio y 340 (36\%) del bajo (37 casos sin información).

Mediciones. La memoria histórica fue pesquisada a través de una pregunta abierta ubicada al inicio del cuestionario: "Mencione los tres eventos de la historia política nacional que usted considera más importantes dentro de los últimos 100 años”. Los encuestadores fueron instruidos a tomar notas textuales de las respuestas dadas por los participantes.

Las respuestas fueron codificadas por dos codificadores independientes, indicando tanto el año de ocurrencia del evento mencionado como la época en que ocurrió. Las épocas fueron definidas a priori: eventos anteriores al siglo XX (que, a pesar de no ser una respuesta formalmente correcta, fue de todos modos usada), eventos del siglo XX previos al gobierno de Allende, gobierno de Allende, golpe de Estado (referidos a los eventos ocurridos el 11 de Septiembre de 1973), dictadura, transición (desde el plebiscito de 1988 hasta el cambio de mando de 1990 en que asume el presidente Aylwin) y eventos actuales (posteriores al cambio de mando de 1990).

La orientación política fue medida con el auto-posicionamiento en una escala de nueve puntos que iba de izquierda (1) a derecha (9), incluyendo el centro (5). Posteriormente clasificamos a los participantes en tres grupos: izquierda (1-3), centro (4-6) y derecha (7-9).

Procedimiento. El cuestionario fue aplicado en el domicilio de los participantes por encuestadores profesionales específicamente capacitados. Los encuestadores informaron a los participantes que sus respuestas serían tratadas de forma confidencial, que su identidad no sería conectada con sus respuestas y que solo se divulgarían resultados de datos agregados.

Análisis. Para comparar las respuestas categorizadas por generaciones y orientación política comparamos la frecuencia de las respuestas categorizadas en épocas para cada grupo usando la prueba $\chi^{2}$ de Pearson con la corrección de Bonferroni. Con esta prueba comparamos si existían diferencias en la frecuencia de las menciones de eventos dentro de una época, entre generaciones y entre grupos políticos.

\section{Resultados}

La orientación política de las personas se distribuyó de la siguiente forma: $186(18,7 \%)$ se identificaron como de izquierda, $494(49,6 \%)$ como de centro y $246(24,7 \%)$ como de derecha. Además, 70 (7\%) entrevistados no se consideraron en ninguna de estas categorías.

Respecto de la categorización de las respuestas sobre memoria histórica en términos del año de ocurrencia del evento mencionado, el $77 \%$ de los participantes indicó en una de las posibles tres menciones un evento que ocurrió en 1973, el año del golpe de Estado, siendo este el año más frecuentemente considerado. En segundo lugar aparece el año 1990, 
con un $27,3 \%$ de los participantes indicando un evento que ocurrió el año del retorno a la democracia. En tercer lugar los participantes mencionaron eventos ocurridos en 1988 $(13,7 \%)$, el año del plebiscito. El año de la elección de Allende, 1970, fue mencionado por el $9,4 \%$ de los participantes. Eventos que ocurrieron el año en que se recolectaron los datos fueron mencionados por un $8,2 \%$ de los participantes. Ninguna otra época alcanzó más de un $6 \%$ de las menciones.

Al comparar la frecuencia con que cada generación mencionó los distintos eventos -en este análisis categorizados en épocas-, observamos que no hay diferencias entre generaciones ni en la primera respuesta, $\chi^{2}(12, N=822)=13,75, p=0,317$, ni en las siguientes dos respuestas, $\chi^{2}(12, N=639)=11,00, p=0,529$, y $\chi^{2}(12, N=488)=9,53$, $p=0,657$, respectivamente. La primera respuesta se concentra en la época del golpe y la segunda y tercera respuestas, en la transición a la democracia (ver Tabla 1).

Tabla 1

Frecuencia de Eventos Mencionados por Época por Cada Generación

\begin{tabular}{|c|c|c|c|c|}
\hline Respuesta & Época & $\begin{array}{c}\text { Jóvenes } \\
(17-21)\end{array}$ & $\begin{array}{c}\text { Adultos } \\
(33-40)\end{array}$ & $\begin{array}{c}\text { Mayores } \\
(50-60)\end{array}$ \\
\hline \multicolumn{5}{|l|}{ Primera respuesta } \\
\hline & Previa al siglo XX & $21(7,5 \%)$ & $20(7,4 \%)$ & $11(4,0 \%)$ \\
\hline & Previa a Allende & $16(5,7 \%)$ & $13(4,8 \%)$ & $17(6,3 \%)$ \\
\hline & Gobierno Allende & $19(6,8 \%)$ & $17(6,3 \%)$ & $16(5,9 \%)$ \\
\hline & Golpe & $188(67,4 \%)$ & $173(63,8 \%)$ & $167(61,4 \%)$ \\
\hline & Dictadura & $6(2,2 \%)$ & $12(4,4 \%)$ & $14(5,1 \%)$ \\
\hline & Transición & $21(7,5 \%)$ & $23(8,5 \%)$ & $30(11,0 \%)$ \\
\hline & Actual & $8(2,9 \%)$ & $13(4,8 \%)$ & $17(6,3 \%)$ \\
\hline & Total & $279(100 \%)$ & $271(100 \%)$ & $272(100 \%)$ \\
\hline \multicolumn{5}{|l|}{ Segunda respuesta } \\
\hline & Previa al siglo XX & $11(5,2 \%)$ & $8(3,9 \%)$ & $9(4,1 \%)$ \\
\hline & Previa a Allende & $14(6,6 \%)$ & $9(4,3 \%)$ & $23(10,4 \%)$ \\
\hline & Gobierno Allende & $15(7,1 \%)$ & $12(5,8 \%)$ & $15(6,8 \%)$ \\
\hline & Golpe & $45(21,3 \%)$ & $46(22,2 \%)$ & $43(19,5 \%)$ \\
\hline & Dictadura & $19(9,0 \%)$ & $22(10,6 \%)$ & $19(8,6 \%)$ \\
\hline & Transición & $86(40,8 \%)$ & $77(37,2 \%)$ & $87(39,4 \%)$ \\
\hline & Actual & $21(10,0 \%)$ & $33(15,9 \%)$ & $25(11,3 \%)$ \\
\hline & Total & $211(100 \%)$ & $207(100 \%)$ & $221(100 \%)$ \\
\hline \multicolumn{5}{|l|}{ Tercera respuesta } \\
\hline & Previa al siglo XX & $9(5,4 \%)$ & $5(3,1 \%)$ & $8(5,0 \%)$ \\
\hline & Previa a Allende & $22(13,2 \%)$ & $17(10,6 \%)$ & $12(7,5 \%)$ \\
\hline & Gobierno Allende & $12(7,2 \%)$ & $7(4,4 \%)$ & $8(5,0 \%)$ \\
\hline & Golpe & $14(8,4 \%)$ & $10(6,3 \%)$ & $18(11,2 \%)$ \\
\hline & Dictadura & $15(9,0 \%)$ & $20(12,5 \%)$ & $18(11,2 \%)$ \\
\hline & Transición & $58(34,7 \%)$ & $56(35,0 \%)$ & $59(36,6 \%)$ \\
\hline & Actual & $37(22,2 \%)$ & $45(28,1 \%)$ & $38(23,6 \%)$ \\
\hline & Total & $167(100 \%)$ & $160(100 \%)$ & $161(100 \%)$ \\
\hline
\end{tabular}

La siguiente comparación que llevamos a cabo se basó en los grupos políticos definidos a partir del auto-posicionamiento en el eje izquierda-derecha. El patrón general es el mismo que en la comparación de generaciones, esto es, no hay diferencias en ninguna de las tres respuestas, $\chi^{2}(12, N=763)=11,13, p=0,518, \chi^{2}(12, N=598)=10,47, p=0,575$ y $\chi^{2}(12$, $N=457)=13,73, p=0,319$, respectivamente. Al igual que en el caso de las generaciones, el golpe de Estado concentra las frecuencias más altas en todos los grupos en la primera 
respuesta, mientras que en la segunda y tercera respuestas existe una mayor frecuencia en las menciones de eventos que ocurrieron en la transición a la democracia (ver Tabla 2).

Tabla 2

Frecuencia de Eventos Mencionados por Época por Cada Grupo Político

\begin{tabular}{|c|c|c|c|c|}
\hline Respuesta & Época & Izquierda & Centro & Derecha \\
\hline \multicolumn{5}{|l|}{ Primera respuesta } \\
\hline & Previa al siglo XX & $7(4,7 \%)$ & $25(6,2 \%)$ & $16(7,7 \%)$ \\
\hline & Previa a Allende & $10(6,7 \%)$ & $22(5,4 \%)$ & $11(5,3 \%)$ \\
\hline & Gobierno Allende & $9(6,0 \%)$ & $29(7,2 \%)$ & $9(4,3 \%)$ \\
\hline & Golpe & $91(60,7 \%)$ & $266(65,7 \%)$ & $136(65,4 \%)$ \\
\hline & Dictadura & $6(4,0 \%)$ & $13(3,2 \%)$ & $8(3,8 \%)$ \\
\hline & Transición & $14(9,3 \%)$ & $37(9,1 \%)$ & $18(8,7 \%)$ \\
\hline & Actual & $13(8,7 \%)$ & $13(3,2 \%)$ & $10(4,8 \%)$ \\
\hline & Total & $150(100 \%)$ & $405(100 \%)$ & $208(100 \%)$ \\
\hline \multicolumn{5}{|l|}{ Segunda respuesta } \\
\hline & Previa al siglo XX & $6(5,3 \%)$ & $11(3,6 \%)$ & $8(4,4 \%)$ \\
\hline & Previa a Allende & $11(9,7 \%)$ & $20(6,6 \%)$ & $14(7,7 \%)$ \\
\hline & Gobierno Allende & $6(5,3 \%)$ & $20(6,6 \%)$ & $13(7,1 \%)$ \\
\hline & Golpe & $28(24,8 \%)$ & $63(20,8 \%)$ & $33(18,1 \%)$ \\
\hline & Dictadura & $5(4,4 \%)$ & $27(8,9 \%)$ & $23(12,6 \%)$ \\
\hline & Transición & $40(35,4 \%)$ & $126(41,6 \%)$ & $67(36,8 \%)$ \\
\hline & Actual & $17(15,0 \%)$ & $36(11,9 \%)$ & $24(13,2 \%)$ \\
\hline & Total & $113(100 \%)$ & $303(100 \%)$ & $182(100 \%)$ \\
\hline \multicolumn{5}{|l|}{ Tercera respuesta } \\
\hline & Previa al siglo XX & $3(3,4 \%)$ & $9(3,8 \%)$ & $9(6,8 \%)$ \\
\hline & Previa a Allende & $11(12,6 \%)$ & $19(8,0 \%)$ & $19(14,3 \%)$ \\
\hline & Gobierno Allende & $6(6,9 \%)$ & $15(6,3 \%)$ & $4(3,0 \%)$ \\
\hline & Golpe & $7(8,0 \%)$ & $21(8,9 \%)$ & $12(9,0 \%)$ \\
\hline & Dictadura & $12(13,8 \%)$ & $22(9,3 \%)$ & $16(12,0 \%)$ \\
\hline & Transición & $25(28,7 \%)$ & $89(37,6 \%)$ & $50(37,6 \%)$ \\
\hline & Actual & $23(26,4 \%)$ & $62(26,2 \%)$ & $23(17,3 \%)$ \\
\hline & Total & $87(100 \%)$ & $237(100 \%)$ & $133(100 \%)$ \\
\hline
\end{tabular}

Los resultados observados confirman en general las hipótesis planteadas, vale decir, existe consenso entre generaciones y grupos políticos respecto de la centralidad del golpe de Estado y la transición a la democracia en la memoria histórica, pero no de eventos específicos que hayan ocurrido durante la dictadura. Ni las diferencias generacionales ni las diferencias en orientación política tienen impacto en la atribución de importancia a eventos específicos para la conformación de la memoria histórica. Estos resultados son estables, incluso al comparar las frecuencias de las menciones en cada una de las tres respuestas dadas por los participantes.

\section{Estudio 2}

\section{Método}

Participantes. En 2006 contactamos a los mismos participantes del Estudio 1, para la segunda medición del estudio de cultura política. De ellos, 695 respondieron un nuevo cuestionario que incluyó una serie de preguntas sobre las políticas de reparación hacia las víctimas de violaciones de los derechos humanos durante la dictadura. Adicionalmente, 
para reemplazar la mortalidad en la muestra, contactamos a 146 personas, siguiendo el mismo método de muestreo y procedimiento que en el Estudio 1, sumando en total 841 participantes. La composición demográfica se mantuvo igual que en el estudio anterior.

Mediciones. Debido a la extensión del cuestionario, las preguntas que usamos son una selección acotada, basada en las escalas originales que tienen una extensión mayor. Elegimos las preguntas en base a su pertinencia conceptual, para maximizar las posibilidades de identificar diferentes dimensiones, y a las propiedades psicométrica de las preguntas, ya sea reportadas en la literatura o en estudios previos.

Orientación política. Fue medida igual que en el Estudio 1.

Agresión autoritaria. Esta variable engloba dos subdimensiones de la escala de autoritarismo de derechas: agresión autoritaria y sumisión autoritaria (Altemeyer, 1981). Múltiples estudios que han usado esta variable han mostrado que es muy difícil distinguir empíricamente las subdimensiones de autoritarismo (ver Funke, 2005). Medimos esta variable con tres preguntas en las que se debía indicar el grado de acuerdo o desacuerdo en una escala de cinco puntos, en la que mayor puntaje indicaba mayor acuerdo, - al igual que en todas las preguntas en los constructos que presentamos a continuación-con las siguientes oraciones: "Los gobiernos deben ocupar mano dura cada vez que hay dificultades", "En vez de tanta preocupación por los derechos de las personas, lo que este país necesita es un gobierno firme" y "Las verdaderas claves para una sociedad exitosa son la obediencia y la disciplina" (ver Haye et al., 2009). La tercera oración corresponde a una medición de sumisión autoritaria.

Convencionalismo. La segunda dimensión de autoritarismo la medimos con cuatro preguntas de acuerdo o desacuerdo: "Las nuevas ideas y estilos de vida están debilitando nuestra sociedad", "Nuestro país estaría mucho mejor si se le diera más importancia a la religión", "Este país tiene problemas porque todos los días están tratando de cambiar algo" y "Este país estaría mejor si se valorara cómo se hacían las cosas en el pasado" (ver Haye et al., 2009).

Orientación a la dominancia social. Pesquisamos esta variable a través de dos preguntas de acuerdo o desacuerdo traducidas de la escala SDO-6 (Sidanius \& Pratto, 1999): "Algunos grupos dentro de nuestro país son simplemente inferiores a otros" y "En este país se le ha dado demasiada importancia al tema de la desigualdad social".

Apoyo a la democracia. Medimos este constructo con dos preguntas (Carvacho \& Haye, 2008): "La democracia es preferible a cualquier otra forma de gobierno" y "A la gente como yo nos da lo mismo un régimen democrático que uno no democrático" (invertido).

Actitudes hacia las politicas de reparación. Para medir este constructo desarrollamos seis preguntas que debían ser respondidas con una de tres categorías: Se ha hecho más de lo conveniente, Se ha hecho lo adecuado y No se ha hecho lo suficiente, a las que se les asignó puntajes de 1 a 3, respectivamente. Las preguntas se refirieron a: "La cantidad de información obtenida acerca de las violaciones a los derechos humanos", "La reparación a las víctimas de las violaciones a los derechos humanos o sus familiares", "El enjuiciamiento a las personas involucradas en la violación a los derechos humanos", "La condena social a las violaciones a los derechos humanos", "La inclusión del tema de los derechos humanos en los textos escolares" y "La preocupación de la opinión pública chilena por este tema”.

Análisis. Llevamos a cabo un modelo de mediación para evaluar el impacto de las actitudes ideológicas que subyacen a la orientación política en las actitudes hacia la reparación. Usamos ecuaciones estructurales, con el estimador de máxima verosimilitud, en la 
versión 5.21 del programa MPlus (Muthén \& Muthén, 2007). Siguiendo las recomendaciones más recientes en la literatura científica para la evaluación de mediaciones, aplicamos un análisis de remuestreo $(k=5000)$ para calcular los efectos indirectos y sus intervalos de confianza con 95\% de seguridad (Hayes, 2009; Rucker, Preacher, Tormala \& Petty, 2011). Los datos perdidos los tratamos usando el método de máxima verosimilitud con información completa.

Los predictores del modelo fueron las actitudes ideológicas de agresión autoritaria y convencionalismo del autoritarismo de derecha (Altemeyer, 1981), orientación a la dominancia social (Pratto et al., 1994; Sidanius \& Pratto, 1999) y apoyo a la democracia (ver Carvacho \& Haye, 2008; Haye et al., 2009). La variable dependiente fue la actitud hacia las políticas de reparación. Calculamos todas estas variables como variables latentes. Las actitudes ideológicas fueron adicionalmente combinadas en un factor latente de segundo orden que captura el núcleo de la configuración ideológica que ordena estas actitudes (Carvacho, 2010). Mayores puntajes en la configuración ideológica se conectan con mayores niveles de derechismo. La orientación política - que en el modelo es la única variable observada- la usamos como mediador.

Utilizamos los siguientes índices de ajuste del modelo: $\chi^{2}$, el índice de ajuste comparativo (CFI), el error cuadrático medio de aproximación (RMSEA) y el residuo cuadrático medio estandarizado (SRMR).

\section{Resultados}

La bondad de ajuste del modelo es apropiada, indicando que representa adecuadamente los datos, $\chi^{2}(129, N=841)=286,74, p<0,001, \mathrm{CFI}=0,96, \mathrm{RMSEA}=0,04$, $\mathrm{SRMR}=0,04$. Todos los pesos factoriales son significativos, $p<0,001$, y sus coeficientes estandarizados son mayores a 0,5 , por lo que la estructura latente del modelo de medición, tanto en primer como en segundo orden, es también apropiada. La Figura 1 muestra todos los coeficientes del modelo.

Los resultados muestran un efecto directo de la orientación política en las actitudes hacia la reparación, $\beta=-0,35, p<0,001$, que indica que mientras los participantes más se orientan a la derecha menos apoyan las políticas de reparación. Adicionalmente se observa un efecto directo de la configuración ideológica en la orientación política, $\beta=0,35, p<0,001$, que confirma que estas actitudes subyacen la orientación política, como ha sido reportado previamente en la literatura (Haye et al., 2009; Jost et al., 2003). A saber, a medida que aumentan los niveles de agresión autoritaria, convencionalismo y de orientación a la dominancia social y disminuye el apoyo a la democracia más se identifican los participantes con la orientación política de derecha.

Con respecto a la hipótesis de mediación, los resultados indican que las actitudes hacia las políticas de reparación son predichas por la configuración ideológica, tanto a través de un efecto directo, $\beta=-0,18, p<0,001,95 \%$ IC $[-0,10,-0,26]$, como a través de un efecto indirecto mediado por la orientación política, $\beta=-0,12, p<0,001,95 \%$ IC $[-0,09,-0,16]$. Los efectos indican que a mayores niveles en la configuración ideológica aumenta la orientación política de derecha, lo que a su vez impacta negativamente en las actitudes hacia las políticas de reparación. Los efectos directos e indirectos de la configuración ideológica más el efecto directo de la orientación política explican un $20 \%, R^{2}=0,20$, de las actitudes hacia las políticas de reparación.

Estos resultados confirman la hipótesis 2 del estudio y se alinean con los reportados por González et al. (en este número), vale decir, las actitudes hacia la reparación varían en función de la orientación política, que a su vez se explica a través de las actitudes ideológicas que diferencian a la derecha y la izquierda. 


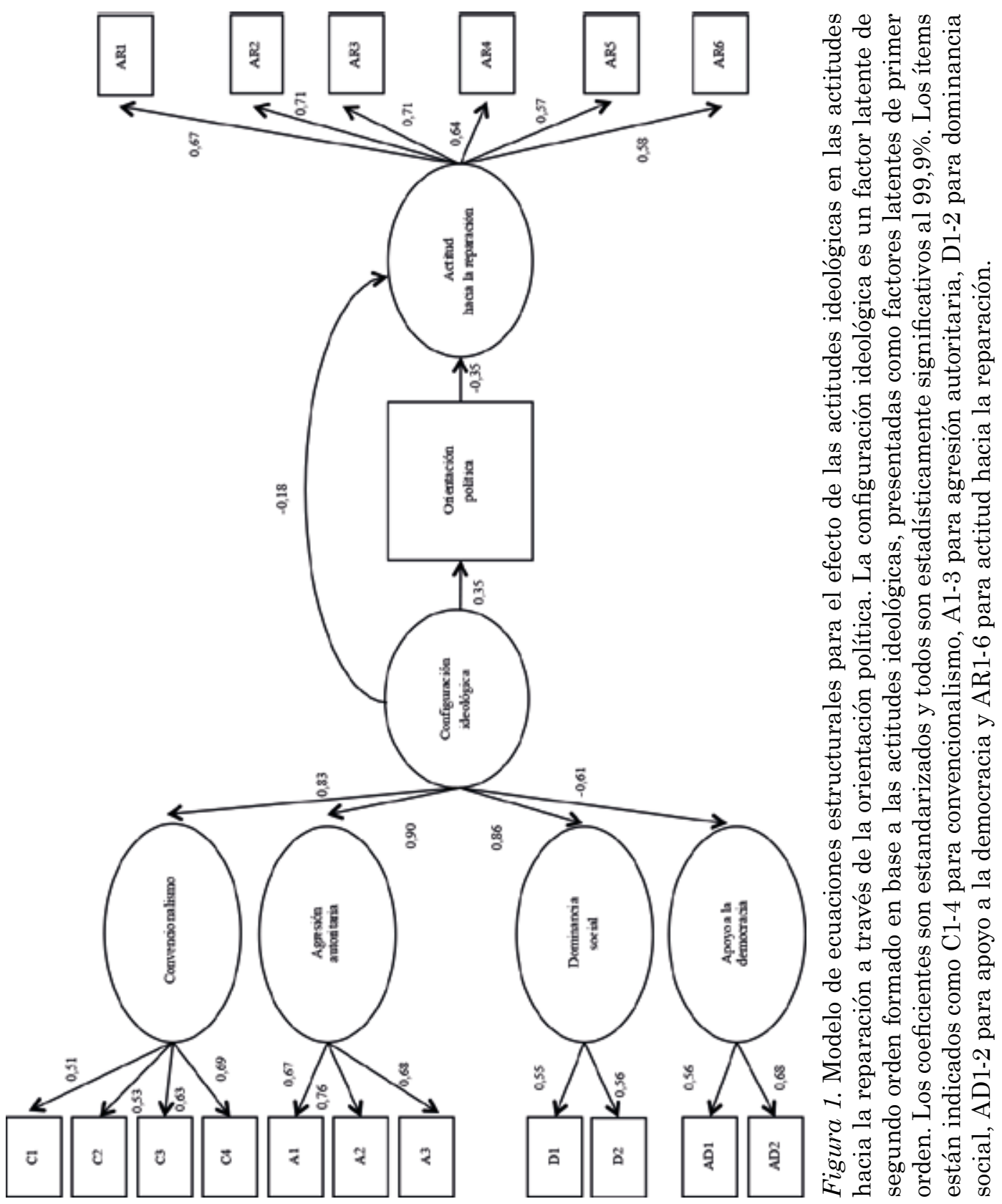




\section{Discusión}

Este artículo aborda el problema del impacto de la dictadura en la conformación de la memoria histórica y en las actitudes hacia las políticas de reparación hacia las víctimas. Abordar este problema adquiere particular importancia al cumplirse 40 años del golpe de Estado de 1973 en Chile. Fechas conmemorativas especiales reactivan las emociones y actitudes que subyacen a los conflictos y llevan a las sociedades a discutir el tema en el contexto de una mirada de futuro que contrapone olvido y memoria (Jelin, 2001). Además, todavía permanecen abiertas múltiples preguntas sobre los procesos de memoria y olvido colectivo. ¿Aumenta el consenso con el paso del tiempo? ¿Aparecen nuevos ámbitos de disenso conectados al pasado traumático? Este artículo buscó dar algunas pistas en esta discusión abierta.

En el contexto de un amplio estudio sobre la cultura política de los chilenos (ver Haye et al., 2009), se llevaron a cabo dos estudios que mostraron que, por un lado, existen visiones consensuales entre generaciones y orientaciones políticas con respecto a la centralidad del golpe de Estado y la transición a la democracia para la construcción de la memoria histórica en Chile. Por otro lado, existe disenso entre orientaciones políticas (izquierda-derecha) con respecto a las actitudes hacia las políticas de reparación hacia las víctimas de la dictadura, siendo las personas de izquierda quienes más apoyan estas políticas. A la diferencia en orientación política subyacen actitudes ideológicas como autoritarismo, orientación a la dominancia social y apoyo a la democracia, que a su vez predicen directa e indirectamente el apoyo a las políticas de reparación. Vale decir, el disenso en las políticas de reparación se explica sustantivamente en función de las actitudes ideológicas. Es importante mencionar que la existencia de un efecto directo de la configuración ideológica, que no es mediado por la orientación política, indica que hay participantes que, a pesar de presentar un patrón de respuestas consistente en términos ideológicos que predice las actitudes hacia la reparación en la forma esperada, no expresan su ideología a través de la auto-identificación en el eje izquierda-derecha. Para aclarar en qué consiste el efecto directo se requieren nuevos estudios que incorporen mediadores adicionales, tales como victimización competitiva (Noor, Shnabel, Halabi \& Nadler, 2012) o emociones (González et al., en este número).

Los resultados de estos estudios expanden la investigación previa sobre las diferencias entre las generaciones en la memoria (e.g., Guichard \& Henríquez, 2011; Manzi et al., 2003), al mostrar en un contexto socio-histórico distinto que el consenso en torno a las relevancia del golpe de Estado y la dictadura es transgeneracional. A su vez, ofrecen evidencia clara de que las actitudes ideológicas que subyacen a la diferenciación entre izquierda y derecha explican también el disenso en las actitudes hacia la reparación, lo cual es un hallazgo novedoso, que expande tanto la investigación sobre actitudes hacia la reparación (Lira \& Morales, 2005; Morales \& Cornejo, 2013) como la investigación sobre las consecuencias de las actitudes ideológicas (e.g., Cárdenas, Meza, Lagues \& Yañez, 2010; Carvacho, 2010; Cohrs, Kämpfe-Hargrave \& Riemann, 2012; Ho et al., 2012).

Es importante también discutir algunas limitaciones del estudio. La primera y más evidente es que la recolección de los datos fue llevada a cabo en los años 2005 y 2006, justamente antes de que se iniciara un evento político post dictadura de tremenda importancia en Chile, a saber, las protestas estudiantiles que adquirieron impacto nacional, primero en 2006, protagonizadas principalmente por estudiantes secundarios, y luego en 2011, incorporando estudiantes secundarios y universitarios con al apoyo de otros actores sociales. $\mathrm{Al}$ momento de diseñar el estudio, pensamos que el marcador generacional de la generación joven del estudio sería la elección presidencial del año 2005, donde resultó electa la presidenta Bachelet. Sin embargo, la aparición del movimiento estudiantil convirtió al grupo más joven de nuestro estudio en una especie de cohorte entre generaciones, pues la nueva generación política parece ser un par de años más joven. 
Una segunda limitación se relaciona con la pregunta del Estudio 1. No podemos garantizar que todos los participantes entendieron la pregunta de la misma forma. No es claro qué entendieron algunos por historia política nacional, lo que se refleja en la mención de hechos evidentemente no políticos (por ejemplo, eventos deportivos). Además, los registros de los encuestadores pueden ser imprecisos, pues no contaron con soporte tecnológico que permitiera asegurar la calidad del registro. Las notas de los encuestadores, a pesar de haber sido capacitados para hacerlo de forma estándar, difieren en su nivel de detalle. Por esta razón, el análisis se enfocó en dos categorizaciones que subsanan el problema de la precisión de los registros (basadas en años y épocas). En el futuro se podrían complementar estos estudios con otros que permitan un mejor registro y preguntas adicionales, de modo de establecer estrategias de análisis que capturen otras facetas de la memoria histórica.

Una tercera limitación es que en este estudio no diferenciamos entre víctimas y no víctimas de la dictadura. Esto es relevante, pues investigaciones previas han mostrado diferencias importantes entre estos dos grupos, en términos de sus emociones y su actitud hacia el perdón y la reparación (Cárdenas, Ascorra, San Martín, Rodríguez \& Páez, 2013; Cárdenas, Páez et al., 2013).

La última limitación es de orden metodológico. A pesar de estar incluido en una investigación de carácter longitudinal, el Estudio 2 utiliza datos transversales y correlacionales para presentar un modelo de mediación con supuestos sobre causalidad en la relación entre las variables. Estos supuestos están basados en la evidencia disponible en la literatura, pero, dado que el modelo presentado es novedoso y original, no han sido evaluados específicamente. Esto se podría resolver utilizando datos longitudinales y modelos que controlen los efectos auto-regresivos.

Finalmente, retomamos las preguntas presentadas al inicio del texto: ¿Qué tan profundo es el impacto de los eventos políticos más importantes de la historia en la cultura política de un país? ¿Es posible hablar de generaciones que son definidas por su experiencia compartida en torno a uno de estos eventos? Este artículo provee evidencia sobre la existencia de una cultura política transgeneracional, en la que el pasado histórico traumático del golpe de Estado y la dictadura sigue constituyendo un campo de diferenciación ideológica fundamental. Esto puede mostrar que en culturas políticas marcadas por altos grados de ideologización, como ha sido la chilena, se pueden producir transmisiones intergeneracionales que en la práctica atenúen o anulen las diferencias generacionales.

\section{Referencias}

Altemeyer, R. A. (1981). Right-wing authoritarianism. Winnipeg, Canadá: University of Manitoba Press.

Alwin, D. F. \& Krosnick, J. A. (1991). Aging, cohorts, and the stability of sociopolitical orientations over the life span. American Journal of Sociology, 97, 169-195. doi:10.1086/229744

Arnoso, M., Cárdenas, M. \& Páez, D. (2012). Diferencias intergeneracionales en la mirada hacia el pasado represivo chileno. Psicología Política, 45, 7-26.

Baltes, P. B. (1987). Theoretical propositions of life-span developmental psychology: On the dynamics between growth and decline. Developmental Psychology, 23, 611-626. doi:10.1037/0012-1649.23.5.611

Baltes, P. B., Staudinger, U. M. \& Lindenberger, U. (1999). Lifespan psychology: Theory and application to intellectual functioning. Annual Review of Psychology, 50, 471-507. doi:10.1146/annurev.psych.50.1.471

Bartlett, F. C. (1932). Remembering: A study in experimental and social psychology. Cambridge, Reino Unido: Cambridge University Press.

Becker, D. \& Díaz, M. (1998). The social process and the transgenerational transmission of trauma in Chile. En Y. Danieli (Ed.), International handbook of multigenerational legacies of trauma (pp. 435-445). New York, NY: Plenum Press.

Cárdenas, M., Ascorra, P., San Martín, M., Rodríguez, M. \& Páez, D. (2013). Emociones como predictores del perdón en el contexto de la violación a los derechos humanos en Chile. Psicoperspectivas: Individuo y Sociedad, 12(1), 30-49.

Cárdenas, M., Meza, P., Lagues, K. \& Yañez, S. (2010). Adaptación y validación de la Escala de Orientación a la Dominancia Social (SDO) en una muestra chilena. Universitas Psychologica, 9, 161-168.

Cárdenas, M., Páez, D. \& Rimé, B. (2013). Transitional justice processes, shared narrative memory about past collective violence and reconciliation. En R. Cabecinhas \& L. Abadia (Eds.), Narratives and social memory: Theoretical and methodological approaches (pp. 61-75). Braga, Portugal: Universidade do Minho. 
Carvacho, H. (2010). Ideological configurations and prediction of attitudes toward immigrants in Chile and Germany. International Journal of Conflict and Violence, 4, 220-233.

Carvacho, H. \& Haye, A. (2008). Configuración ideológica y estructura social: resucitando el tema desde la psicología política. Revista de Psicología Universidad de Chile, 17(2), 81-94. doi:10.5354/07190581.2008 .17138

Cohrs, J. C., Kämpfe-Hargrave, N. \& Riemann, R. (2012). Individual differences in ideological attitudes and prejudice: Evidence from peer-report data. Journal of Personality and Social Psychology, 103, 343-361. doi:10.1037/a0028706

Colomer, J. M. \& Escatel, L. E. (2005). La dimensión izquierda-derecha en América Latina. Desarrollo Económico, 45, 123-136.

Concha, V., Guichard, E. \& Henríquez, G. (2009). Memoria histórica y paradigma del curso de vida. Investigación CEVI-Chile. Sociedad Hoy, 17, 51-61.

Cornejo, M., Brackelaire, J. L. \& Mendoza, F. (2009). Des chaînes du silence à la chaîne de l'écoute [De las cadenas del silencio a la cadena de la escucha]. Cahiers de Psychologie Clinique, 32, 203-231. doi:10.3917/ cpc.032.0203

Cornejo, M., Morales, G., Kovalskys, J. \& Sharim, D. (2013). La escucha de la tortura desde el Estado: la experiencia de los profesionales de la Comisión Nacional Sobre Prisión Política y Tortura en Chile. Universitas Psychologica, 12, 271-284.

Cornejo, M., Rojas, R., Buzzoni, M. E., Mendoza, F., Concha, M. \& Cabach, C. (2007). Prisión política y tortura: desde las intervenciones psicosociales a las políticas de reparación. Persona y Sociedad, 21(1), 59-81.

Cornejo, M., Rojas, R. C. \& Mendoza, F. (2009). From testimony to life story: The experience of professionals in the Chilean National Commission on Political Imprisonment and Torture. Peace and Conflict: Journal of Peace Psychology, 15, 111-133. doi:10.1080/10781910802603450

Escobar, M. \& Fernández, R. (2008). Performatividad, memoria y conmemoración: la experiencia de la marchaRearme en el Chile post-dictatorial. Forum: Qualitative Social Research, 9(2), Artículo 36.

Faúndez, X. \& Cornejo, M. (2010). Aproximaciones al estudio de la transmisión transgeneracional del trauma psicosocial. Revista de Psicología Universidad de Chile, 19(2), 31-54. doi:10.5354/0719-0581.2010.17107

Funke, F. (2005). The dimensionality of right-wing authoritarianism: Lessons from the dilemma between theory and measurement. Political Psychology, 26, 195-218. doi:10.1111/j.1467-9221.2005.00415.x

Guichard, E. \& Henríquez, G. (2011). Memoria histórica en Chile: una perspectiva intergeneracional desde Concepción. Reis: Revista Española de Investigaciones Sociológicas, 135, 3-26. doi:10.5477/cis/reis.135.3

Halbwachs, M. (1925). Les cadres sociaux de la mémoire [Los marcos sociales de la memoria]. Paris, Francia: Albin Michel.

Haye, A. (2003). Collective memory: An investigation into its cognitive and group processes (Tesis de Doctorado no publicada), The University of Sheffield, Reino Unido.

Haye, A., Carvacho, H., González, R., Manzi, J. \& Segovia, C. (2009). Relación entre orientación política y condición socioeconómica en la cultura política chilena: una aproximación desde la psicología política. Polis: Revista de la Universidad Bolivariana, 23, 351-384.

Hayes, A. F. (2009). Beyond Baron and Kenny: Statistical mediation analysis in the new millennium. Communication Monographs, 76, 408-420. doi:10.1080/03637750903310360

Ho, A. K., Sidanius, J., Pratto, F., Levin, S., Thomsen, L., Kteily, N. \& Sheehy-Skeffington, J. (2012). Social dominance orientation: Revisiting the structure and function of a variable predicting social and political attitudes. Personality and Social Psychology Bulletin, 38, 583-606. doi:10.1177/0146167211432765

Jelin, E. (2001). Exclusión, memorias y luchas políticas. En D. Mato (Comp.), Estudios latinoamericanos sobre cultura y transformaciones sociales en tiempos de globalización (pp. 91-110). Buenos Aires, Argentina: Consejo Latinoamericano de Ciencias Sociales.

Jost, J. T. (2006). The end of the end of ideology. American Psychologist, 61, 651-670. doi:10.1037/0003066X.61.7.651

Jost, J. T., Federico, C. M. \& Napier, J. L. (2009). Political ideology: Its structure, functions, and elective affinities. Annual Review of Psychology, 60, 307-337. doi:10.1146/annurev.psych.60.110707.163600

Jost, J. T., Glaser, J., Kruglanski, A. W. \& Sulloway, F. J. (2003). Political conservatism as motivated social cognition. Psychological Bulletin, 129, 339-375. doi:10.1037/0033-2909.129.3.339

Kovalskys, J. \& Morales, G. (2001). Violencia política, trauma y su impacto en los procesos psíquicos de la adolescencia. Psykhe, 10(2), 47-52.

Lira, E., Becker, D. \& Castillo, M. I. (1991). Derechos humanos: todo es según el dolor con que se mira. Santiago, Chile: Instituto Latinoamericano de Salud Mental y Derechos Humanos.

Lira, E. \& Castillo, M. I. (1991). Psicología de la amenaza política y del miedo. Santiago, Chile: Instituto Latinoamericano de Salud Mental y Derechos Humanos.

Lira, E. \& Morales, G. (Eds.) (2005). Derechos humanos y reparación: una discusión pendiente. Santiago, Chile: LOM.

Mannheim, K. (1952). The problems of generations. En K. Mannheim (Ed.), Essays on the sociology of knowledge (pp. 276-322). London, Reino Unido: Routledge \& Kegan Paul.

Manzi, J., Helsper, E., Ruiz, S., Krause, M. \& Kronmüller, E. (2003). El pasado que nos pesa: la memoria colectiva del 11 de Septiembre de 1973. Revista de Ciencia Politica, 23(2), 177-214. doi:10.4067/S0718090X2003000200009

Manzi, J., Ruiz, S., Krause, M., Meneses, A., Haye, A. \& Kronmüller, E. (2004). Memoria colectiva del golpe de Estado de 1973 en Chile. Revista Interamericana de Psicología, 38, 153-169. 
Mavor, K. I., Louis, W. R. \& Laythe, B. (2011). Religion, prejudice, and authoritarianism: Is RWA a boon or bane to the psychology of religion? Journal for the Scientific Study of Religion, 50, 22-43. doi:10.1111/j.1468$5906.2010 .01550 . \mathrm{x}$

Mayer, K. U. (2009). New directions in life course research. Annual Review of Sociology, 35, 413-433. doi:10.1146/ annurev.soc.34.040507.134619

McFarland, S. (2010a). Authoritarianism, social dominance, and other roots of generalized prejudice. Political Psychology, 31, 453-477. doi:10.1111/j.1467-9221.2010.00765.x

McFarland, S. (2010b). Personality and support for universal human rights: A review and test of a structural model. Journal of Personality, 78, 1735-1764. doi:10.1111/j.1467-6494.2010.00668.x

Montenegro, M. \& Piper, I. (2009). Reconciliación y construcción de la categoría víctima: implicaciones para la acción política en Chile. Revista de Psicología Universidad de Chile, 18(1), 31-60. doi:10.5354/07190581.2009 .17126

Morales, G. \& Cornejo, M. (2013). Ambivalencias en la escucha de la tortura en Chile: implicancias clínicas y sociales. Terapia Psicológica, 31(2), 197-208.

Muthén, L. K. \& Muthén, B. O. (2007). Mplus user's guide (5 $5^{a}$ versión). Los Angeles, CA: Autores.

Noor, M., Shnabel, N., Halabi, S. \& Nadler, A. (2012). When suffering begets suffering: The psychology of competitive victimhood between adversarial groups in violent conflicts. Personality and Social Psychology Review, 16, 351-374. doi:10.1177/1088868312440048

Osborne, D., Sears, D. O. \& Valentino, N. A. (2011). The end of the solidly democratic south: The impressionableyears hypothesis. Political Psychology, 32, 81-108. doi:10.1111/j.1467-9221.2010.00796.x

Piper, I. (2005). Obstinaciones de la memoria: la dictadura militar chilena en las tramas del recuerdo (Tesis de Doctorado no publicada), Departamento de Psicología Social, Universidad Autónoma de Barcelona, España.

Prado, M. I. \& Krause, M. (2004). Representaciones sociales de los chilenos acerca del 11 de Septiembre de 1973 y su relación con la convivencia cotidiana y con la identidad chilena. Psykhe, 13(2), 57-72. doi:10.4067/ S0718-22282004000200005

Pratto, F., Sidanius, J., Stallworth, L. M. \& Malle, B. F. (1994). Social dominance orientation: A personality variable predicting social and political attitudes. Journal of Personality and Social Psychology, 67, 741-763. doi:10.1037/0022-3514.67.4.741

Reyes, M. J. (2007). El pasado reciente en el Chile de hoy: entre la reconciliación y la convivencia. Persona y Sociedad, 21(1), 39-58.

Rucker, D. D., Preacher, K. J., Tormala, Z. L. \& Petty, R. E. (2011). Mediation analysis in social psychology: Current practices and new recommendations. Social \& Personality Psychology Compass, 5, 359-371. doi:10.1111/j.1751-9004.2011.00355.x

Ruiz, M. S. \& Krause, M. (2003). Memorias colectivas y reconciliación nacional en Chile: a treinta años del golpe militar. Persona y Sociedad, 22(3), 221-247.

Sahdra, B. \& Ross, M. (2007). Group identification and historical memory. Personality and Social Psychology Bulletin, 33, 384-395. doi:10.1177/0146167206296103

Schuman, H. \& Corning, A. D. (2006). Comparing Iraq to Vietnam: Recognition, recall, and the nature of cohort effects. Public Opinion Quarterly, 70, 78-87. doi:10.1093/poq/nfj010

Schuman, H. \& Rodgers, W. L. (2004). Cohorts, chronology, and collective memories. Public Opinion Quarterly, 68, 217-254. doi:10.1093/poq/nfh012

Schuman, H. \& Scott, J. (1989). Generations and collective memories. American Sociological Review, 54, 359381. doi:10.2307/2095611

Sears, D. O. \& Valentino, N. A. (1997). Politics matters: Political events as catalysts for preadult socialization. American Political Science Review, 91, 45-65. doi:10.2307/2952258

Sharim, D., Kovalskys, J., Morales, G. \& Cornejo, M. (2011). Trauma psicosocial y memoria: diseño de un dispositivo biográfico para investigar el impacto de la Comisión de Prisión Política y Tortura en Chile. Revista de Estudios Sociales, 40, 81-88. doi:10.7440/res40.2011.08

Sidanius, J. \& Pratto, F. (1999). Social dominance: An intergroup theory of social hierarchies and oppression. Cambridge, Reino Unido: Cambridge University Press.

Sidanius, J. \& Pratto, F. (2012). Social dominance theory. En P. A. M. Van Lange, A. W. Kruglanski \& E. T. Higgins (Eds.), Handbook of theories of social psychology (Vol. 2, pp. 418-438). London, Reino Unido: Sage. doi:10.1348/014466603322127193

Tocornal, X. (2008). The Chilean memory debate: Mapping the language of polarisation (Tesis de Doctorado no publicada), Loughborough University, Reino Unido.

Toro, S. (2008). De lo épico a lo cotidiano: jóvenes y generaciones políticas en Chile. Revista de Ciencia Política, 28(3), 143-160. doi:10.4067/S0718-090X2008000200006

Valentino, N. A. \& Sears, D. O. (1998). Event-driven political communication and the preadult socialization of partisanship. Political Behavior, 20, 127-154. doi:10.1023/A:1024880713245

Waldman, G. (2009). Chile: la persistencia de las memorias antagónicas. Política y Cultura, 31, $211-234$.

Fecha de recepción: Marzo de 2013.

Fecha de aceptación: Julio de 2013. 